\title{
Good news about a bad subject: scientific evidence to help defeat multidrug/ extensively drug-resistant tuberculosis
}

\author{
Giovanni Sotgiu and Mark Woodhead ${ }^{2,3}$
}

\begin{abstract}
Affiliations: ${ }^{1}$ Clinical Epidemiology and Medical Statistics Unit, Dept of Biomedical Sciences, University of Sassari - Research, Medical Education and Professional Development Unit, AOU Sassari, Italy. ${ }^{2}$ Faculty of Medical and Human Sciences, University of Manchester, Manchester, UK. ${ }^{3}$ Dept of Respiratory Medicine, Central Manchester University Hospitals NHS Foundation Trust, Manchester Academic Health Science Centre, Manchester, UK.
\end{abstract}

Correspondence: Mark Woodhead, Dept of Respiratory Medicine, Manchester Royal Hospital, Infirmary Road, Manchester, M13 9WL, UK. E-mail: mark.woodheaddacmft.nhs.uk

@ERSpublications

This editorial describes, and puts into context, the statement on M/XDR-TB written by the TBNET Group http://ow.ly/wFoxr

The good news about tuberculosis (TB) is that in the 29 reporting European Union/European Economic Area countries the number of incident TB cases is falling, with an average annual decline of $6 \%$ since 2011 [1]. The bad news is that, however you look at it, multidrug-resistant TB (MDR-TB) and extensively drugresistant TB (XDR-TB) remain a major clinical and public health problem worldwide, particularly in former Soviet Union countries and Central Asia [2-4]. Less than a quarter of the total estimated MDR-TB cases were actually diagnosed in 2012 [2], with dramatic consequences from an individual and a community perspective (i.e. increased morbidity, mortality and drug-resistant Mycobacterium tuberculosis transmission). It was estimated that 450000 incident patients developed MDR-TB and 170000 died from MDR-TB. More than half of such cases die, fail or stop taking treatment and only one in three cases in reporting countries completes the long treatment course ( $>20$ months). The treatment success in the 2010 MDR-TB cohort was $48 \%$, only $34(31.8 \%)$ out of 107 countries reached a therapeutic success rate of at least $75 \%$. About $9.6 \%$ of the MDR-TB cohort was XDR-TB. The direct and indirect costs are significant from a healthcare and a societal perspective $[5,6]$. While the cost per case for drug-susceptible TB is estimated to be $\sim € 10000$, that for MDR-TB and for XDR-TB is estimated to be $\sim € 50000$ and $€ 170000$, respectively [5]. The reasons for this increased cost are fairly obvious since successful management often requires a prolonged inpatient stay, exposure to a cocktail of multiple, expensive, usually less effective medications with frequent serious and unpleasant toxicities, to be taken under close medical and/or social supervision for many months (so-called directly observed therapy). That a significant proportion of initially drugsusceptible TB can become MDR during treatment, following poor patient adherence mainly caused by drug toxicity and long treatment duration, is also a concern [7-9]. Case management is not helped by the geographical distribution of TB patients across countries. For instance, of the 54 World Health Organization (WHO) European Region countries reporting 33371 cases of MDR-TB in 2012, five reported more than 1000 individuals with TB annually (Belarus, Kazakhstan, Russia, Ukraine and Uzbekistan), while 20 countries reported less than 10 individuals annually. Clinical experience in MDR-TB management is thus concentrated in few, sometimes poorly resourced countries, whereas cases are thinly spread across other better resourced countries. Guidance on MDR-TB management is, therefore, welcome to most healthcare workers involved in TB patient care and the consensus statement produced by the Tuberculosis Network European Trials Group (TBNET) group in this issue of the European Respiratory Journal delivers exactly what is needed [10].

Received: May 022014 | Accepted: May 022014

Conflict of interest: None declared.

Copyright @ERS 2014 
The document is made up of 21 different sections citing 267 references. After the introduction, methods and definitions section it describes the epidemiology of M/XDR-TB in Europe and risk factors associated with their occurrence. Sections on contact tracing, infection control and the interpretation of resistance testing results follow. The extensive treatment sections cover drug and surgical management, duration, adverse events, and sections on special considerations including in- and outpatient care, children and pregnancy. Further sections deal with management in the presence of HIV co-infection, consilium (an individual patient-directed multidisciplinary approach based on the recommendations of TB experts) and palliative care before final sections covering consensus statements and future priorities. It is, thus, both a mammoth and a comprehensive effort from the 40 authors, providing a mine of detailed information for anyone involved in the management of M/XDR-TB.

The document covers a lot of material already available in other publications for example from the WHO [11-13]. In this respect it is disappointing that in many areas it adds little new to the information already available in such documents. This is especially the case around the topic of newly licensed drugs and the optimal duration of therapy, which are two clinical areas where guidance is often most needed. The recommendation to use a panel of experts (i.e. a consilium) to guide individual patient therapy, using for instance remote access via the internet, is to be welcomed as a potential model of care, especially in lowincidence countries; however, an evidence base to show that treatment outcomes are improved by this approach is currently absent [14].

Although this manuscript can be deemed a monumental work collecting the opinions of TB specialists in several fields, the main Achilles' heel is the methodology adopted by the authors: the scientific evidence is not systematically retrieved, following internationally agreed upon methodological guidelines, but based on the experts' opinion [15-18]. Some missing scientific documents could be important to give a comprehensive overview on the abovementioned specific topics. Consequently, the strength of the recommendations could be not so relevant and potentially biased. However, the inclusion of statements issued by international governmental organisations, such as WHO and European Centre for Disease Prevention and Control, can partially overcome the methodological shortcomings, giving reliability to the recommendations' section. In addition, high quality evidence, on which to base recommendations for many of the questions that arise in M/XDR-TB management, is lacking in the medical literature.

The TBNET manuscript can be considered a summary of what has been done to date, potentially drafting the new research frontiers for the forthcoming years.

The M/XDR-TB phenomenon, as demonstrated by the relevant work performed by the TBNET authors, is characterised by multiple features and complications that should be approached in a multi-specialty and multidisciplinary manner. It is relatively novel but a slow, poorly-funded, less-focused research approach was implemented relative to that performed globally when the HIV/AIDS epidemic started. If the current diagnostic, therapeutic and preventive tools were deemed insufficient to fight pan-susceptible TB, their inappropriateness is striking in the face of M/XDR-TB. Following this "wait and see" overture the epidemiological indicators related to $\mathrm{M} / \mathrm{XDR}-\mathrm{TB}$, and consequently those related to $\mathrm{TB}$, are getting worse.

A strong, more target-oriented political commitment is needed at international, national and sub-national level, involving not only the stakeholders of the healthcare world, but also those societies, communities and individuals of the economic and social worlds, which can play a crucial role in the multi-sectorial conflict against drug-resistant TB [19].

As clearly, directly and indirectly, demonstrated in the TBNET paper, numerous aspects should be controlled to avoid the forecasted catastrophic evolution of M/XDR-TB. The aetiopathogenesis of this serious worldwide medical event does not reside only in the mismanagement of drug-susceptible TB and in the poor infection control strategies of the drug-resistant cases, but also in the serious global economic crisis (since the 1980s TB incidence has been positively correlated to poverty) [20, 21], in the social inequalities (total health coverage, inadequate and imbalanced access to healthcare services, stigmatisation of the disease for patients and their families in the workplace and in the community, etc.) [20,21], and in the TB research and development gaps [22, 23].

Therefore, new global efforts are needed, with special attention given to deprived geographical areas where the incidence of M/XDR-TB is increasing favoured by a changeable, unpredictable, poor, unsatisfactory healthcare, social, economic and political context.

Perhaps, in future documents, it would be important to better focus the concentration on some specific hot topics needing an urgent solution from the clinicians and the public health experts (e.g. palliative care of individuals with few and inadequate therapeutic options or management of the contacts of the XDR-TB patients, particularly of some categories at high risk of TB disease, such as HIV-positives or children). 
It is straightforward that systematic, high-quality, scientific evidence is needed for this emerging issue. It should be used to create an operational agenda of healthcare and research needs, adequately detected worldwide, as a starting point of all future basic, translational, and clinical activities.

Only with a strong scientific evidence base is it possible to build new policies, strategies, and recommendations, which will have to be adopted and adapted to the different epidemiological, demographic, social and economic scenarios.

In the recent past, variable clinical approaches and public health policies have favoured procedural shortcomings that promoted the emergence and spread of drug-resistant M. tuberculosis strains [9]. Education and training of public healthcare providers, as well as of communities, civil society, academics, private healthcare practitioners, industry and other relevant stakeholders is fundamental. All of them should be intensively involved in a common effort guided by high quality-evidence based strategies [19]. However, the role of governments has to be central: they should increase the capacity for detecting M/XDR-TB patients in association with the capacity for delivering high-quality anti-TB drugs and high-quality continuous care in both hospital and community settings [2, 19].

The new WHO post-2015 strategy, targeted to the elimination of TB by 2050 (i.e. $<1$ contagious case per 1000000 inhabitants), will only be successful if there is a specific political and scientific commitment, fighting TB comprehensively [19]. M/XDR-TB could represent a dramatic nightmare if we continue to use the same old diagnostic, therapeutic, and preventive weapons. The summary of information on M/XDR-TB in the TBNET consensus statement is a helpful start, but it is only the start!

\section{References}

1 European Centre for Disease Prevention and Control, World Health Organization Regional Office for Europe. Tuberculosis surveillance and monitoring in Europe 2014. Stockholm, European Centre for Disease Prevention and Control, 2014

2 World Health Organization. Global tuberculosis report 2013. WHO/HTM/TB/2013.11. Geneva, WHO Press, 2013.

3 Migliori GB, Loddenkemper R, Blasi F, et al. 125 years after Robert Koch's discovery of the tubercle bacillus: the new XDR-TB threat. Is "science" enough to tackle the epidemic? Eur Respir J 2007; 29: 423-427.

4 Migliori GB, Sotgiu G, Lange C, et al. Extensively drug-resistant tuberculosis: back to the future. Eur Respir J 2010; 36: 475-477.

5 Diel R, Vandeputte J, de Vries G, et al. Costs of tuberculosis disease in the European Union: a systematic analysis and cost calculation. Eur Respir J 2014; 43: 554-565.

6 Tanimura T, Jaramillo E, Weil D, et al. Financial burden for tuberculosis patients in low- and middle-income countries: a systematic review. Eur Respir J 2014; 43: 1763-1775.

7 Jenkins HE, Crudu V, Soltan V, et al. High risk and rapid appearance of multidrug resistance during tuberculosis treatment in Moldova. Eur Respir J 2014; 43: 1132-1141.

8 Caminero JA. Multidrug-resistant tuberculosis: epidemiology, risk factors and case finding. Int J Tuberc Lung Dis 2010; 14: 382-390.

9 Migliori GB, Sotgiu G, D'Ambrosio L, et al. TB and MDR/XDR-TB in European Union and European Economic Area countries: managed or mismanaged? Eur Respir J 2012; 39: 619-625.

10 Lange C, Abubakar I, Alffenaar J-WC, et al. Management of patients with multidrugresistant/extensively drugresistant tuberculosis in Europe: a TBNET consensus statement. Eur Respir J 2014; 43: 23-63.

11 World Health Organization. Guidelines for the programmatic management of drug-resistant tuberculosis. Emergency update 2008. WHO/HTM/TB/2008.402. Geneva, WHO Press, 2008.

12 Falzon D, Jaramillo E, Schünemann HJ, et al. WHO guidelines for the programmatic management of drug-resistant tuberculosis: 2011 update. Eur Respir J 2011; 38: 516-528.

13 Migliori GB, Zellweger JP, Abubakar I, et al. European union standards for tuberculosis care. Eur Respir J 2012; 39: 807-819.

14 D'Ambrosio L, Tadolini M, Dupasquier S, et al. ERS/WHO Tuberculosis Consilium: reporting of the initial 10 cases. Eur Respir J 2014; 43: 286-289.

15 Jaeschke R, Guyatt GH, Dellinger P, et al. Use of GRADE grid to reach decisions on clinical practice guidelines when consensus is elusive. BMJ 2008; 337: a744.

16 Guyatt GH, Oxman AD, Kunz R, et al. Going from evidence to recommendations. BMJ 2008; 336: 1049-1051.

17 Karanicolas PJ, Kunz R, Guyatt GH. Point: evidence-based medicine has a sound scientific base. Chest 2008; 133: 1067-1071.

18 Guyatt GH, Oxman AD, Kunz R, et al. What is "quality of evidence" and why is it important to clinicians? BMJ 2008; 336: 995-998.

19 World Health Organisation. Global strategy and targets for tuberculosis prevention, care and control after 2015. http://apps.who.int/gb/ebwha/pdf_files/EB134/B134_12-en.pdf Date last updated: November 29, 2013. Date last accessed: May 12, 2014.

20 Spence DP, Hotchkiss J, Williams CS, et al. Tuberculosis and poverty. BMJ 1993; 307: 759-761.

21 Hawker JI, Bakhshi SS, Ali S, et al. Ecological analysis of ethnic differences in relation between tuberculosis and poverty. BMJ 1999; 319: 1031-1034.

22 Lienhardt C. Fundamental research is the key to eliminating TB. Nature 2014; 507: 401.

23 Lienhardt C, Espinal M, Pai M, et al. What research is needed to stop TB? Introducing the TB Research Movement. PLoS Med 2011; 8: e1001135. 